\title{
Perigee attitude maneuvers of geostationary satellites during electric orbit raising
}

DOI:

10.2514/1.G002370

\section{Document Version}

Accepted author manuscript

Link to publication record in Manchester Research Explorer

\section{Citation for published version (APA):}

Mostaza Prieto, D., \& Roberts, P. (2017). Perigee attitude maneuvers of geostationary satellites during electric orbit raising. Journal of Guidance, Control, and Dynamics, 40(8), 1978-1989. https://doi.org/10.2514/1.G002370

\section{Published in:}

Journal of Guidance, Control, and Dynamics

\section{Citing this paper}

Please note that where the full-text provided on Manchester Research Explorer is the Author Accepted Manuscript or Proof version this may differ from the final Published version. If citing, it is advised that you check and use the publisher's definitive version.

\section{General rights}

Copyright and moral rights for the publications made accessible in the Research Explorer are retained by the authors and/or other copyright owners and it is a condition of accessing publications that users recognise and abide by the legal requirements associated with these rights.

\section{Takedown policy}

If you believe that this document breaches copyright please refer to the University of Manchester's Takedown Procedures [http://man.ac.uk/04Y6Bo] or contact uml.scholarlycommunications@manchester.ac.uk providing relevant details, so we can investigate your claim.

\section{OPEN ACCESS}




\title{
Perigee attitude maneuvers of geostationary
}

\section{satellites during electric orbit raising}

\author{
David Mostaza-Prieto ${ }^{1}$ and Peter C.E. Roberts ${ }^{2}$ \\ The University of Manchester, Manchester, England M13 9PL, United Kingdom
}

When full-electric orbit raising trajectories begin in a classic geostationary transfer orbit with low initial perigee altitude, the need for deployed solar arrays to power the propulsion system significantly increases the aerodynamic and gravity gradient torques. In fact, the torque magnitudes in the first few perigee passages may become a challenging requirement for the attitude control system. Apart from oversizing actuators, other solutions may include the need for a backup thruster system or raising the perigee altitude, implying mass penalties and cost. This paper presents the design of an optimal attitude maneuver at the perigee that can be undertaken using nominal reaction wheels. Attitude paths avoiding saturation of the wheels while dumping accumulated momentum are obtained performing a physically consistent modelling of aerodynamic torques and using Pseudospectal methods to solve the trajectory optimization problem. The optimization of solar array positions is also explored to further constrain the problem or improve the maneuver performance. Resulting mass and cost savings can be significant, which could be used for additional payload or to significantly extend the operational life of the satellite.

\footnotetext{
${ }^{1}$ Researcher, School of Mechanical, Aerospace and Civil Engineering, The University of Manchester, Manchester, England M13 9PL, United Kingdom.

${ }^{2}$ Lecturer, School of Mechanical, Aerospace and Civil Engineering, The University of Manchester, Manchester, England M13 9PL, United Kingdom.
} 


\section{Nomenclature}

$\boldsymbol{A}=$ rotation matrix

$C_{l}=$ roll aerodynamic moment coefficient

$C_{m}=$ pitch aerodynamic moment coefficient

$C_{n}=$ yaw aerodynamic moment coefficient

$\boldsymbol{h}=$ momentum vector

$I \quad=$ inertia matrix, $k g-m^{2}$

$J \quad=$ cost function

$L_{r e f}=$ aerodynamic reference length, $m$

$\boldsymbol{r}=$ position vector, $m$

$S_{r e f}=$ aerodynamic reference area, $m^{2}$

$\boldsymbol{T}=$ external torques vector, N.m

$\boldsymbol{v} \quad=$ velocity vector, $\mathrm{m} / \mathrm{s}$

$V_{a}=$ aerodynamic velocity, $\mathrm{m} / \mathrm{s}$

$\alpha \quad=$ angle of attack, deg

$\alpha_{a c c}=$ accommodation coefficient

$\beta \quad=$ angle of sideslip, deg

$\delta \quad=$ solar array position, degrees

$\mu \quad=$ cost function

$\rho \quad=$ atmospheric density, $\mathrm{kg} / \mathrm{m}^{3}$

$\boldsymbol{\sigma}=$ Modified Rodrigues Parameters vector

$\boldsymbol{\omega} \quad=$ angular rate, $\mathrm{rad} / \mathrm{s}$

\section{Introduction}

Full-electric orbit raising of geostationary Earth orbit (GEO) platforms has become a reality [1-4]. Electric propulsion technology is mature enough and it is widely accepted that it may drive the future of low cost access to space [5-7]. In fact, almost all the GEO satellite manufacturers offer this option in their current designs [6]. The high specific impulse of the low thrust engines allows 
for great mass savings at the expense of longer transfer times, of the order of months. During the transfer, the electric engine is on most of the time and the spacecraft is constantly changing its attitude in order to point the thrust vector into the optimal direction. Therefore, the realization of such long trajectories imposes new requirements in systems such as mission analysis [8, 9], guidance, navigation, propulsion [6], or spacecraft shielding due to space weather exposure [10].

One of the issues arising from the use of standard geostationary transfer orbits (GTO) is the low altitude of initial perigees. With the exception of Proton, most of the currently available launchers, Falcon 9, Atlas V or Delta IV, place the satellite into an injection orbit with the nominal perigee altitude at $185 \mathrm{~km}$ [11-13]. Ariane V GTO has a perigee of $250 \mathrm{~km}$. [14]. At these altitudes the Earth's atmosphere has a noticeable effect, and the torques resulting from the impingement of atmospheric particles on satellite surfaces are significant.

For typical GEO spacecraft, the perigee passage does not impose an important requirement on the attitude and control system. They employ bi-propellant thrusters for attitude control, which have enough control authority to overcome the disturbance torques at the perigee. Furthermore, during the early orbit phases many platforms remain with the solar arrays stowed or half stowed, minimizing the magnitude of aerodynamic torques. Finally, there are no special attitude requirements at the perigee, so the satellite undertakes the passage inertially pointed.

In contrast, full-electric platforms lack a bi-propellant attitude control thruster system. Also, in order to provide enough power to the electric propulsion system, the solar arrays need to be deployed at the beginning of the mission. For the case of an injection into low Earth orbit (LEO), the resulting drag may reduce considerably the effective thrust, a problem that has been addressed in $[15,16]$. For a GTO this is not a concern, however, the deployed arrays can create a considerable aerodynamic torque at perigee. In addition, depending on the initial orbit, the electric orbit thrust profile may require the satellite to be in a near velocity pointing attitude in the vicinity of the perigee. Therefore, an attitude maneuver may be needed during the perigee passage to move the satellite from that attitude required before the perigee, to that attitude required after the perigee. Although such platforms can be equipped with a cold gas thruster system, its usage may not be possible or desirable once the arrays are deployed or the electric propulsion system is functioning. 
Moreover, it requires propellant usage and therefore imposes a mass penalty. In such cases the only available actuators are the reaction wheels. However, the combination of limited momentum capacity with the high torque environment may quickly saturate the wheels when performing a classic eigenaxis attitude maneuver.

One way to overcome this problem is by increasing the altitude of the perigee with the launcher so to avoid the effects of aerodynamic drag. Although some launch vehicles allow it, in practice, this is a de-optimization of the injection orbit and a non-negligible mass penalty is associated. As a reference, using public domain data for a major launch vehicle, Ariane IV, the payload mass was reduced by $1.2 \mathrm{~kg}$ if the perigee was increased $1 \mathrm{~km}$ above the nominal [17]. Therefore, a performance impact of - $204 \mathrm{~kg}$ was needed to raise the perigee altitude from $180 \mathrm{~km}$ to $350 \mathrm{~km}$. For an electric satellite, this is equivalent to several years of in-orbit station-keeping (SK) [9]. Assuming an average Isp of $2000 \mathrm{~s}$, a $\Delta V$ budget of $50 \mathrm{~m} / \mathrm{s} /$ year for $\mathrm{SK}$ and and an initial in-orbit mass ranging from 2000 to $4000 \mathrm{~kg}$, the mass consumed per year ranges from 5 to $10 \mathrm{~kg}$. Therefore, a typical 15-year mission would require 75 to $150 \mathrm{~kg}$ of propellant for SK. Although there is usually limited availability of new launch vehicle performance specifications, the same principles and orders of magnitude would apply.

In this paper, we propose an alternative solution without any associated cost. An optimized attitude maneuver can be implemented at the perigee to cope with the significant gravity and aerodynamic torques. Rather than following the shortest path between two attitudes while fighting against naturally occurring environmental torques, the attitude path can take advantage of them in order to prevent wheel saturation. Essentially, a physically consistent modelling of disturbance torques allows their use in an advantageous way. This concept is known as zero propellant maneuver (ZPM) [18], owing to the possibility of maneuvering a spacecraft without the need for propellant to desaturate the reaction wheels. Using pseudospectral (PS) methods for the solution of the optimal guidance problem [19-21], Bedrossian et al. successfully applied this approach to several slews of 90 and 180 degrees in the International Space Station without saturation of its CMGs [22-24]. PS methods have been proven to be an efficient and robust way to provide solutions to complex continuous-time optimal control problems [25], like the one that arises from the formulation of the 
ZPM. Some other reported solutions to optimal attitude guidance problems by means of PS methods are the flight tested time-optimal slews on the TRACE spacecraft $[26,27]$, or the development of torque optimal guidance algorithms for cubesats [28].

We apply this optimal maneuver concept to the previously presented problem of a satellite equipped with just reaction wheels, maneuvering in the perigee vicinity of a geostationary transfer orbit in order to follow the guided trajectory of an electric orbit raising satellite. The solution is obtained with MATLAB by means of PS methods using a combination of codes: Gauss pseudospectral optimization software (GPOPS) [29] and the non-linear programming (NLP) solver provided in the numerical algorithms group (NAG) toolbox [30].

As highlighted above, during the perigee passage aerodynamic torques become the main disturbance torques, therefore, a comprehensive modelling of the aerodynamic torques and potential uncertainties is performed. We also incorporate the novel idea of optimising the solar array motion between two given positions, allowing improved performance of the maneuver. Different cost functions such as actuator usage or solar array aerodynamic load are explored. Finally, we show that these maneuvers can also be used to dump considerable amount of momentum at perigee, or to relax momentum wheel requirements.

\section{Equations of motion}

The equations of motion are derived in the local vertical local horizontal frame (LVLH) where the $z$-axis points towards the center of the earth, $y$ is perpendicular to the orbit plane, in the direction of the negative orbit normal, and $x$ completes the right hand side orthogonal frame. We use Modified Rodrigues Parameters (MRP) for attitude parametrization, Eq. 1. This set of parameters describes any rotation below 360 degrees without singularities [31]. At the same time they have the advantage to provide a minimal parametrization. For this problem the expected rotations are below $360 \mathrm{deg}$, so MRP representation is advantageous compared to quaternions, which require an extra parameter.

$$
\boldsymbol{\sigma}=\left[\begin{array}{l}
\sigma_{1} \\
\sigma_{2} \\
\sigma_{3}
\end{array}\right]
$$


The attitude kinematic equation of the MRP with respect to LVLH is given by [32]:

$$
\begin{aligned}
& \dot{\boldsymbol{\sigma}}=\boldsymbol{Q}(\boldsymbol{\sigma})\left(\left.\boldsymbol{\omega}_{b i}\right|_{b}-\left.\boldsymbol{\omega}_{r i}\right|_{b}\right) \\
& \boldsymbol{Q}(\boldsymbol{\sigma})=\left[\begin{array}{ccc}
1-\sigma_{1}^{2}-\sigma_{2}^{2}-\sigma_{3}^{2} & 2\left(\sigma_{1} \sigma_{2}-\sigma_{3}\right) & 2\left(\sigma_{1} \sigma_{3}+\sigma_{2}\right) \\
2\left(\sigma_{1} \sigma_{2}+\sigma_{3}\right) & 1-\sigma_{1}^{2}+\sigma_{2}^{2}-\sigma_{3}^{2} & 2\left(\sigma_{2} \sigma_{3}-\sigma_{1}\right) \\
2\left(\sigma_{1} \sigma_{3}-\sigma_{2}\right) & 2\left(\sigma_{2} \sigma_{3}+\sigma_{1}\right) & 1-\sigma_{1}^{2}-\sigma_{2}^{2}+\sigma_{3}^{2}
\end{array}\right]
\end{aligned}
$$

The vectors $\left.\boldsymbol{\omega}_{b i}\right|_{b}$ and $\left.\boldsymbol{\omega}_{r i}\right|_{b}$ are the angular velocities of the body, $b$, and LVLH, $r$, frames with respect to inertial frame, expressed in body frame. The derivation $\left.\boldsymbol{\omega}_{r i}\right|_{b}$ for a general non-circular orbit can be found in [33]:

$$
\begin{gathered}
\left.\boldsymbol{\omega}_{r i}\right|_{b}=\left.\boldsymbol{A}_{b r}(\boldsymbol{\sigma}) \boldsymbol{\omega}_{r i}\right|_{r} \\
\left.\boldsymbol{\omega}_{r i}\right|_{r}=\left[\begin{array}{c}
0 \\
-\omega_{j} \\
0
\end{array}\right] \\
\omega_{j}=\frac{-1}{r^{2}|\boldsymbol{v} \times \boldsymbol{r}|} \boldsymbol{v} \cdot\left[(\boldsymbol{r} \cdot \boldsymbol{v}) \boldsymbol{r}-r^{2} \boldsymbol{v}\right]
\end{gathered}
$$

where $\boldsymbol{A}_{b r}$ is the transformation matrix from LVLH frame to body given by Eq. 7 and, $\boldsymbol{r}$ and $\boldsymbol{v}$ are the orbit position and velocity vectors.

$$
\boldsymbol{A}_{b r}(\boldsymbol{\sigma})=\frac{1}{\left(1+\boldsymbol{\sigma}^{\top} \boldsymbol{\sigma}\right)^{2}}\left[\begin{array}{ccc}
4\left(\sigma_{1}^{2}-\sigma_{2}^{2}-\sigma_{3}^{2}\right)+\Sigma^{2} & 8 \sigma_{1} \sigma_{2}+4 \sigma_{3} \Sigma & 8 \sigma_{1} \sigma_{3}-4 \sigma_{2} \Sigma \\
8 \sigma_{1} \sigma_{2}-4 \sigma_{3} \Sigma & 4\left(-\sigma_{1}^{2}+\sigma_{2}^{2}-\sigma_{3}^{2}\right)+\Sigma^{2} & 8 \sigma_{3} \sigma_{2}+4 \sigma_{1} \Sigma \\
8 \sigma_{1} \sigma_{3}+4 \sigma_{2} \Sigma & 8 \sigma_{3} \sigma_{2}-4 \sigma_{1} \Sigma & 4\left(-\sigma_{1}^{2}-\sigma_{2}^{2}+\sigma_{3}^{2}\right)+\Sigma^{2}
\end{array}\right]
$$

Then, the dynamic equation of motion for a satellite equipped with reaction wheels is [33]:

$$
\boldsymbol{T}=\left.\dot{\boldsymbol{h}}\right|_{i}=\left.\dot{\boldsymbol{h}}\right|_{b}+\boldsymbol{\omega}_{b i} \times\left.\boldsymbol{h}\right|_{b}
$$

where $\boldsymbol{h}$ is the momentum of the system, that can be divided into momentum of the rigid body, $\boldsymbol{h}_{s c}$, and momentum stored in the wheels, $\boldsymbol{h}_{w}$.

$$
\boldsymbol{h}=\boldsymbol{h}_{s c}+\boldsymbol{h}_{w}=I \boldsymbol{\omega}_{b i}+\boldsymbol{h}_{w}
$$


For this problem, external torques, $\boldsymbol{T}$ are made up of gravity gradient and aerodynamic contributions:

$$
\boldsymbol{T}=\boldsymbol{T}_{g g}(t)+\boldsymbol{T}_{a}(t)
$$

Then, substituting in Eq. 9:

$$
I \dot{\boldsymbol{\omega}}_{b i}=\boldsymbol{T}_{g g}(t)+\boldsymbol{T}_{a}(t)-\dot{\boldsymbol{h}}_{w}-\boldsymbol{\omega}_{b i} \times\left(I \boldsymbol{\omega}_{b i}+\boldsymbol{h}_{w}\right)
$$

where $I$ is the satellite inertia matrix. The configuration and number of reaction wheels is different for each platform, for the general case of $n$ reaction wheels:

$$
\boldsymbol{h}_{w}=\sum_{i=1}^{n} \boldsymbol{a}_{i} h_{w, i}
$$

where $\boldsymbol{a}_{i}$ is the vector giving the orientation of the spin axis of the wheel $i$ with respect to the satellite body frame. Then, the angular momentum of each individual wheel is:

$$
h_{w, i}=I_{w, i}\left(\Omega_{w, i}+\boldsymbol{a}_{i}^{\top} \boldsymbol{\omega}_{b i}\right)
$$

In the previous equation $I_{w, i}$ is the wheel inertia about the spin axis, and $\Omega_{w, i}$ the angular rate of the wheel. Finally, the torque applied to the wheel axis by the motor equals the rate of change of the wheel momentum:

$$
\dot{h}_{w, i}=\tau_{w, i}
$$

\section{A. Environmental torques}

The geometry of the satellite is shown in Fig. 1. It is modeled as a central box with two deployed solar arrays. Table 1 presents the main geometric values and inertia. During a perigee passage, the two main external torque contributors are the gravity gradient torque and the aerodynamic torque. The gravity gradient torque results from the non-uniform mass distribution of the satellite. Its expression in body axes is given by:

$$
\boldsymbol{T}_{g g}(t)=\frac{3 \mu}{r^{3}} \boldsymbol{A}_{b r}(\boldsymbol{\sigma})_{3} \times\left(I \boldsymbol{A}_{b r}(\boldsymbol{\sigma})_{3}\right)
$$

The aerodynamic torque results from the friction of the upper atmosphere with the exposed satellite surfaces. At satellite altitudes the atmosphere cannot be modeled as a continuous fluid and Free 


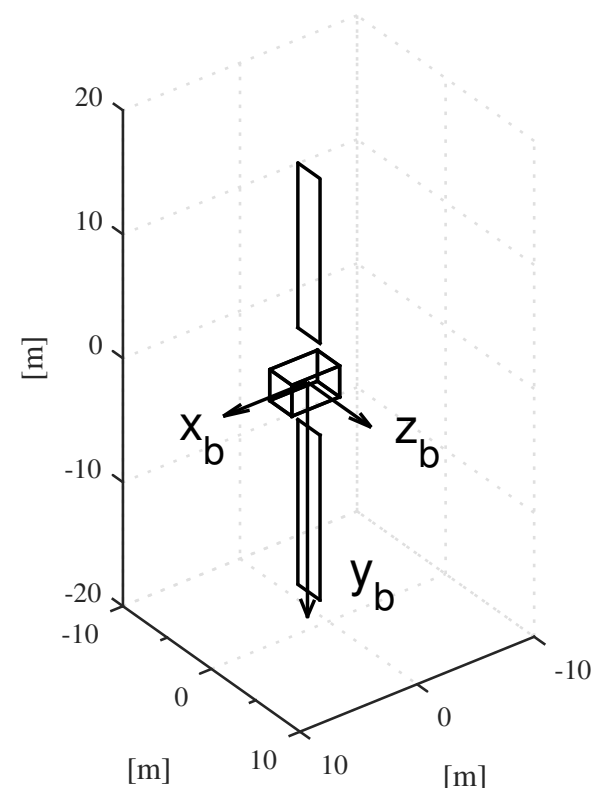

Fig. 1: Representation of a geostationary satellite and body frame

Molecular Flow (FMF) has to be assumed. The common expression of aerodynamic torque is:

$$
\boldsymbol{T}_{a}(t)=\frac{1}{2} \rho(t) V_{a}(t)^{2} S_{r e f} L_{r e f}\left[\begin{array}{c}
C_{l}^{G} \\
C_{m}^{G} \\
C_{n}^{G}
\end{array}\right]
$$

where $\rho$ represents the atmospheric density, $V_{a}$ is the relative velocity magnitude of the vehicle with respect to the atmosphere, $S_{r e f}$ and $L_{r e f}$ are the reference surface and length and $C_{l}^{G}, C_{m}^{G}$ and $C_{n}^{G}$ are the rolling, pitching and yawing coefficients already translated to CoG. The determination of aerodynamic torques represents the largest uncertainty to the problem. Therefore, a careful study needs to be performed in order to obtain physically representative values. For instance, the density has to be predicted utilizing a high altitude atmospheric model, examples of which are NRLMESISE00 [34] or JB2008 [35]. Depending on solar and geomagnetic activity, the magnitude of atmospheric density can vary significantly, similarly affecting the magnitude of the aerodynamic torques. However, modern density models, like the ones mentioned, are able to take these variations into account. Relative velocity with respect to the flow is calculated assuming the atmosphere to be co-rotating with the Earth. There exist as well high altitude winds [36], that modify the direction and magnitude of the relative velocity. However, for this problem, inertial velocity at perigee is large enough $(9-10 \mathrm{~km} / \mathrm{s})$ so high altitude winds $(10-200 \mathrm{~m} / \mathrm{s})$ can be neglected. Finally, 
Table 1: Satellite geometric properties

\begin{tabular}{lcc}
\hline \hline & & Units \\
\hline Center of Gravity (CoG) & {$[1.9,-0.02,0.03]$} & $m$ \\
Array Surface & 32 & $\mathrm{~m}^{2}$ \\
Array 1 CoG position & {$[1.6,-10,0]$} & $\mathrm{m}$ \\
Array 2 CoG position & {$[1.6,10,0]$} & $\mathrm{m}$ \\
Inertia & {$[26000,6000,23000]$} & $\mathrm{kg} / \mathrm{m}^{2}$ \\
\hline
\end{tabular}

the aerodynamic coefficients in Free Molecular Flow are a function of satellite geometry, incidence angle with respect to the flow (angle of attack, $\alpha$, and sideslip, $\beta$ ), and gas-surface interaction. Several models and modelling techniques are available [37]. The main difficulty and uncertainty is the determination of the gas-surface interaction, which is the same as predicting the mean speed and trajectories of the gas particles after colliding with the surface. The two limiting cases to describe the angular distribution of the reemitted particles are the diffuse reflection, characteristic of contaminated surfaces, and the quasi specular reflection, where the molecules are reemitted in a narrow beam close to the specular angle, characteristic of clean surfaces (Fig. 2). The other parameter used in many models is the accommodation coefficient, $\alpha_{a c c}$ [38], which is the ratio of the mean kinetic energy the reemitted particles have lost after the collision, to the mean kinetic energy loss in a full accommodated collision. That means if the particles are reemitted with the energy corresponding to the surface temperature, $E_{w}$ :

$$
\alpha_{a c c}=\frac{E_{i}-E_{r}}{E_{i}-E_{w}}
$$

where $E_{i}$ is the incident kinetic energy and $E_{r}$ is the actual mean kinetic energy of the reemitted particles. Exposed surfaces of satellites in LEO orbits have been discovered to be contaminated with atomic oxygen [39]. This fact results in highly diffuse distribution of remitted particles (97\%) together with high accommodation values (0.9). However, for a GTO, this assumption needs to be revised. The higher energy collisions due to the speed at perigee together with the less dwelling time for contamination, decreases the accommodation of the impinging particles. This has been experimentally measured by Moe for a GTO, where using a Sentman model [40] (assuming complete 


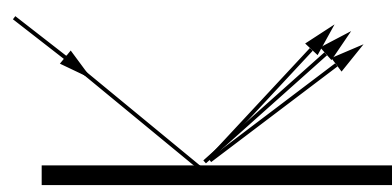

quasi - specular

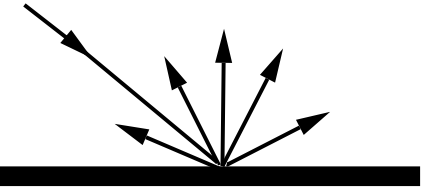

diffuse

Fig. 2: Quasi specular (left) and diffuse (right) reflections

diffuse reflection), accommodation coefficients of only 0.67 were obtained. Although some degree of specular reflections may take place, they conclude that the majority of particles are reflected in a diffuse manner, otherwise the obtained accommodation coefficients are physically unrealistic. Following these results, we use in this study a complete diffuse Sentman model with $\alpha_{a c c}=0.7$. Then, the aerodynamic coefficients can be expressed as:

$$
\begin{aligned}
& C_{l}^{G}=C_{l}^{G}\left(\alpha(t), \beta(t), \boldsymbol{\delta}(t), T_{w}, \alpha_{a c c}, s(t)\right) \\
& C_{m}^{G}=C_{l}^{G}\left(\alpha(t), \beta(t), \boldsymbol{\delta}(t), T_{w}, \alpha_{a c c}, s(t)\right) \\
& C_{n}^{G}=C_{l}^{G}\left(\alpha(t), \beta(t), \boldsymbol{\delta}(t), T_{w}, \alpha_{a c c}, s(t)\right)
\end{aligned}
$$

where $s(t)$ represents the molecular speed ratio, defined as the ratio of thermal speed to bulk velocity, $T_{w}$ is the satellite wall temperature, and $\boldsymbol{\delta}=\left(\delta_{1}, \delta_{2}\right)$ the positions of the upper and lower solar arrays, they have a large impact on the satellite geometry and hence the resulting aerodynamic torques. The aerodynamic database for $\alpha_{a c c}=0.7$ is shown in Fig. 3. The temperature of the satellite surface is assumed to be $300 \mathrm{~K}$ and the speed ratio $s=7$. Fig. 4 shows $C_{m}$ values for zero angle of attack and sideslip and different lower solar array positions. The 0 degrees position corresponds to the array normal parallel to the x-body.

Finally, it is important to understand the limitations and uncertainties of the aerodynamic model. Fig. 6 shows, for the test GEO satellite of Fig. 1, the uncertainty created by the accommodation coefficient on the yaw aerodynamic coefficient $C_{n}^{G}$ at different side slip angles and zero angle of attack. The amount of uncertainty depends on satellite geometry and center of gravity position. Also, Fig. 7 presents the uncertainty created by the amount of specular component, the higher the altitude the more specular component is to be expected together with lower accommodation 


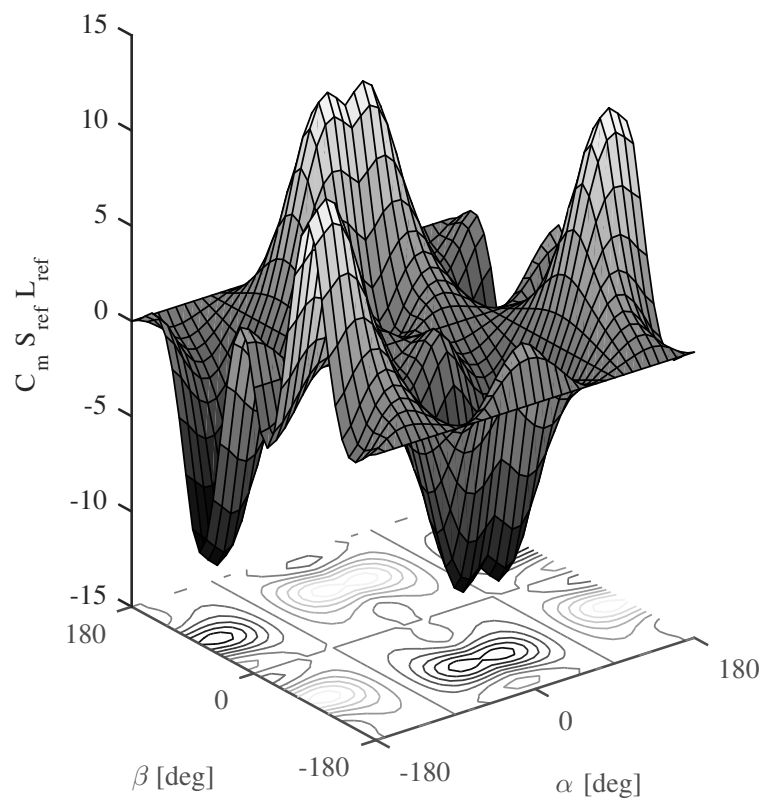

Yaw coefficient

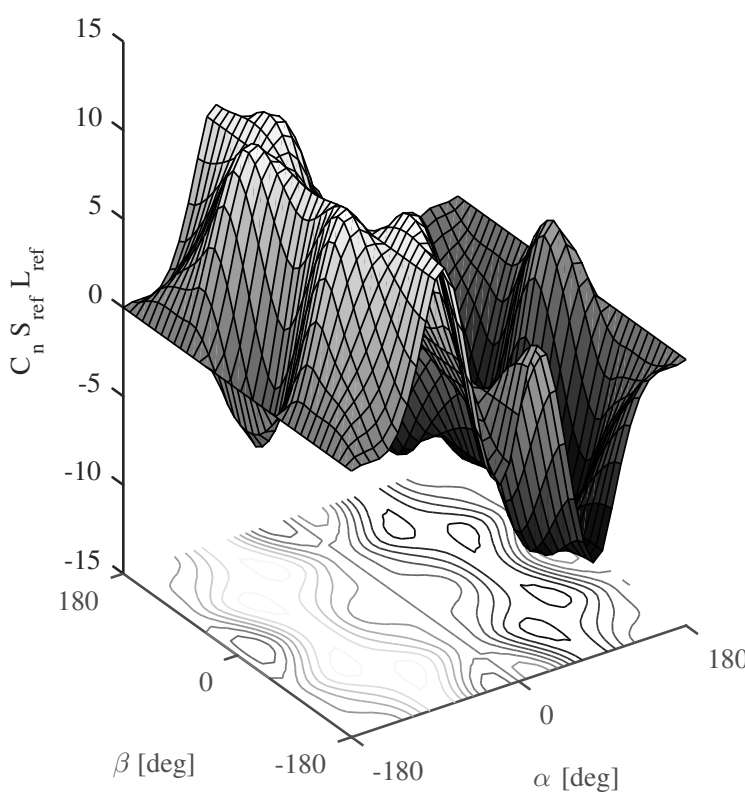

Fig. 3: Aerodynamic coefficients for the satellite of Fig. 1, obtained with a Sentman model

coefficients. As a final comment, the FMF closed-form solutions may be in the limit of applicability $(K n>10)$ during a small region around the exact perigee point (Fig. 5 for high solar activity). Below $150 \mathrm{~km}$ the collisions between particles start to be important and the accuracy of analytical FMF solutions decreases [41]. The quantification of this effect requires the use of computationally intensive numerical codes, like Direct Simulation Monte Carlo (DSMC) [42]. For the considered altitude, the associated uncertainty is probably lower than the uncertainty due to accommodation coefficient or specular reflection component. 

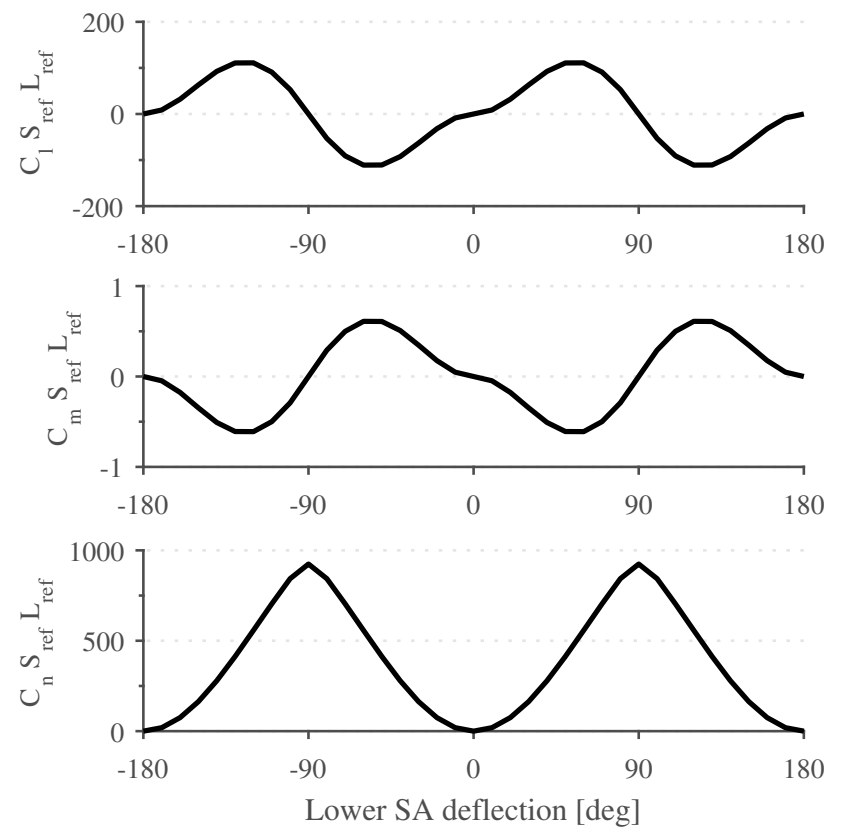

Fig. 4: Variation of aerodynamic coefficients with the position of the lower solar array, obtained with a Sentman model for $\alpha=\beta=0$

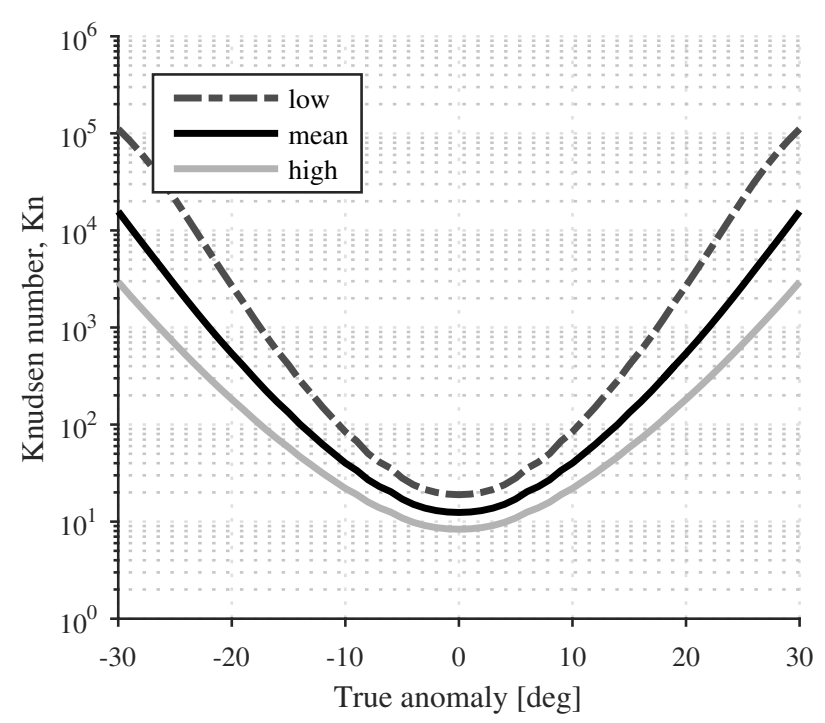

Fig. 5: Knudsen number around the perigee of a GTO orbit for high, low and mean solar activity.

Perigee altitude $=185 \mathrm{~km}$ and characteristic length $=10 \mathrm{~m}$. 


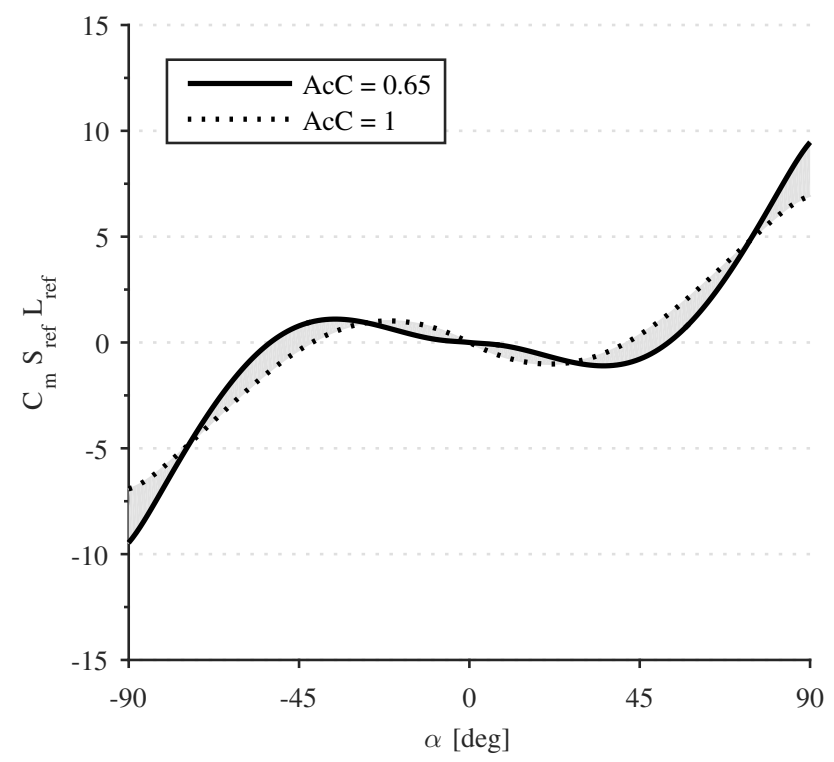

Fig. 6: Uncertainty produced by the accommodation coefficient

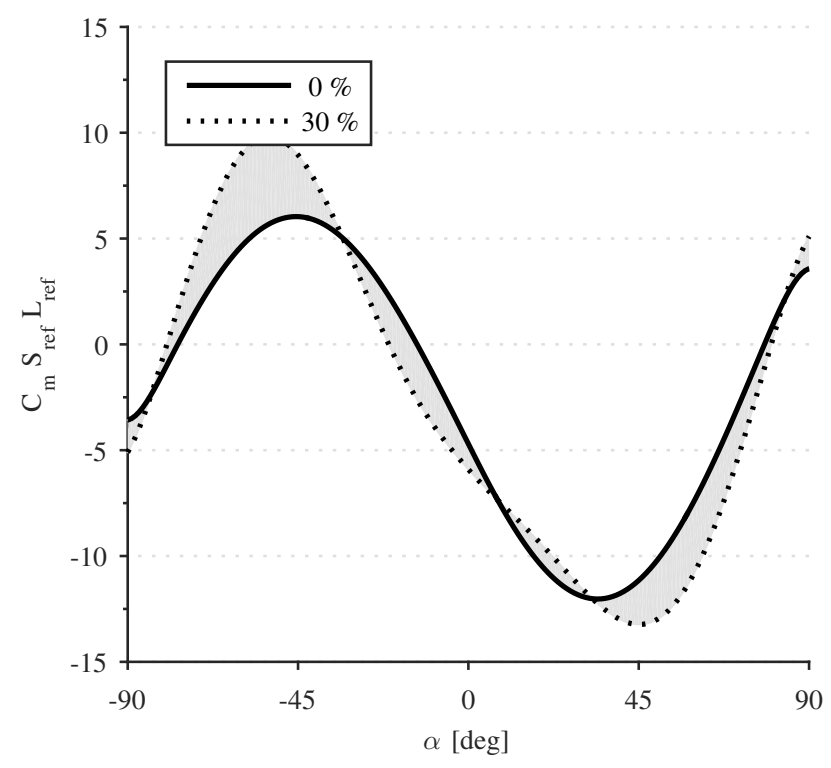

Fig. 7: Uncertainty produced by the amount of specular component, $0 \%$ corresponds to full diffuse reflections 


\section{Optimal Control Problem}

The goal is to transfer the satellite from an initial state before the perigee, $\boldsymbol{x}\left(t_{0}\right)$, composed of attitude, rate, initial momentum stored in the wheels and solar array position, to a given final state after the perigee, $\boldsymbol{x}\left(t_{f}\right)$ (Fig. 8), without exceeding the momentum capacity of the reaction wheels (path constraint). In order to simplify and generalize, we assume that the satellite is equipped with three reaction wheels aligned with the body axes. The solar arrays are initially sun-pointed, and should rotate in body axes so to remain sun-pointed at the final attitude. In between these two points the satellite is free to perform an attitude maneuver subjected to given constraints. Different attitude trajectories produce different profiles of wheel momentum. For those ones fulfilling the constraints, we can decide which one is more optimal by defining a certain quantity to be minimized, the cost function. The formulation of this optimal control problem then becomes:

$$
\begin{aligned}
& \text { Minimize } \quad J\left[\boldsymbol{x}(\cdot), \boldsymbol{u}(\cdot), t_{f}\right]=\int_{0}^{t_{f}} \boldsymbol{h}_{w}^{\top} \boldsymbol{h}_{w} d t \\
& \text { subject to } \dot{\boldsymbol{x}}(t)=\left[\begin{array}{c}
\boldsymbol{v}(t) \\
-\mu \frac{\boldsymbol{r}(t)}{r(t)^{3}} \\
\boldsymbol{Q}(\boldsymbol{\sigma})\left(\left.\boldsymbol{\omega}_{b i}\right|_{b}-\left.\boldsymbol{\omega}_{r i}\right|_{b}\right) \\
J^{-1}\left(\boldsymbol{T}_{g g}(t)+\boldsymbol{T}_{a}(t, \boldsymbol{\delta})-\dot{\boldsymbol{h}}_{w}(t)+\boldsymbol{\omega}_{b i} \times\left(I \boldsymbol{\omega}_{b i}+\boldsymbol{h}_{w}(t)\right)\right) \\
\boldsymbol{u}(t) \\
\frac{\boldsymbol{\delta}_{0}-\boldsymbol{\delta}_{f}}{t_{f}-t_{0}}
\end{array}\right] \\
& \boldsymbol{x}\left(t_{0}\right)=\left(\boldsymbol{r}_{0}, \boldsymbol{v}_{0}, \boldsymbol{\sigma}_{0}, \boldsymbol{\omega}_{0}, \boldsymbol{h}_{w 0}, \boldsymbol{\delta}_{0}\right) \\
& \boldsymbol{x}\left(t_{f}\right)=\left(\boldsymbol{r}_{F}, \boldsymbol{v}_{F}, \boldsymbol{\sigma}_{F}, \boldsymbol{\omega}_{F}, \boldsymbol{h}_{w F}, \boldsymbol{\delta}_{F}\right) \\
& \left|\omega_{i}(t)\right| \leq \omega_{M A X} \quad i=1,2,3 \\
& \left|h_{w i}(t)\right| \leq h_{w M A X} \quad i=1,2,3 \\
& \left|u_{i}(t)\right| \leq \tau_{M A X} \quad i=1,2,3
\end{aligned}
$$

where the state and control spaces are given by:

$$
\begin{aligned}
& \mathbb{X}:=\left\{\left(\boldsymbol{r}, \boldsymbol{v}, \boldsymbol{\sigma}, \boldsymbol{\omega}, \boldsymbol{h}_{w}, \boldsymbol{\delta}\right) \in \mathbb{R}^{17}:\|\boldsymbol{\omega}\|_{\infty}<\omega_{M A X},\left\|\boldsymbol{h}_{w}\right\|_{\infty} \leq h_{w M A X}\right\} \\
& \mathbb{U}:=\left\{\boldsymbol{u} \in \mathbb{R}^{3}:\|\boldsymbol{u}\|_{\infty} \leq \tau_{M A X}\right\}
\end{aligned}
$$

In Eq. 22, $\boldsymbol{u}(t)$ is the optimal control torque applied around the reaction wheel spin axis, 


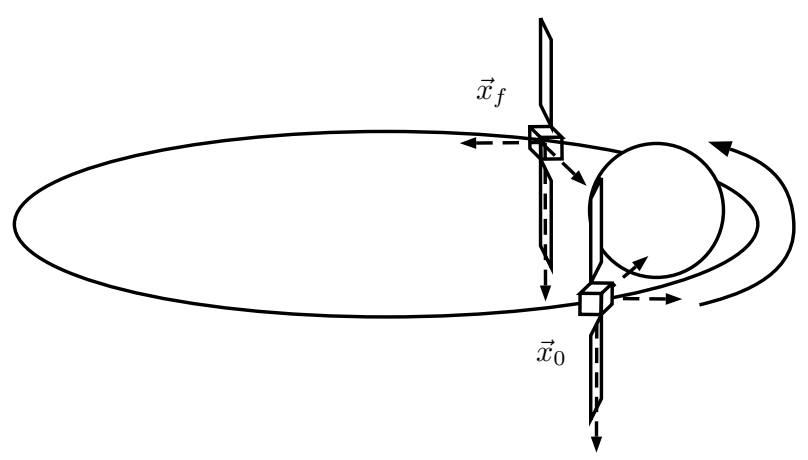

Fig. 8: Approximate geometry of the optimization problem

$\tau_{M A X}$ is the available maximum torque delivered by the wheels and $h_{w M A X}$ is the maximum wheel capacity. Notice that a fixed rotation rate, $\dot{\boldsymbol{\delta}}=\frac{\boldsymbol{\delta}_{0}-\boldsymbol{\delta}_{f}}{t_{f}-t_{0}}$, is commanded to the arrays (initial and final attitudes are known together with flight time, $t_{f}$ ). We can also constrain the final array position and give the arrays the freedom to move between initial and final states. This way gives the problem an extra degree of freedom allowing to improve performance, or include more constraints. Then, the $\dot{\boldsymbol{\delta}}$ equation is replaced by:

$$
\dot{\boldsymbol{\delta}}=\boldsymbol{u}_{2}(t)
$$

and

$$
\mathbb{U}:=\left\{\left(\boldsymbol{u}_{1}, \boldsymbol{u}_{2}\right) \in \mathbb{R}^{5}:\left\|\boldsymbol{u}_{1}\right\|_{\infty} \leq \tau_{M A X},\left\|\boldsymbol{u}_{2}\right\|_{\infty} \leq \Omega_{S A}\right\}
$$

where $\boldsymbol{u}_{2}(t)$ is the vector containing the two rate commands for each of the arrays. The following path constraint needs to be also specified in such cases, where $\Omega_{S A}$ is the maximum rate the solar array drive mechanism can deliver.

$$
\left|u_{2 i}(t)\right| \leq \Omega_{S A} \quad i=1,2
$$

In this case, the moments of inertia of the solar arrays and the torques necessary to change the rotation rate would need to be included in the equations. However, we will ignore them for simplicity. Regarding the cost function, multiple choices are possible. For instance, a fairly common choice are those cost functions referring to actuator usage, either by minimizing the peak of momentum magnitude, or by minimizing the integral cost of actuation (torque applied to the wheels). In this 
paper we choose to minimize the integral cost of accumulated wheel momentum.

$$
J_{1}=\int_{0}^{t_{f}} \boldsymbol{h}_{w}^{\top} \boldsymbol{h}_{w} d t
$$

Another interesting cost function is the minimization of aerodynamic loads on the solar arrays, which is roughly equivalent to minimising the drag force on the panels.

$$
J_{2}=\int_{0}^{t_{f}} C_{D}^{S A}
$$

Other cost functions could be the minimization of depointing from a certain attitude or the minimization of the aerodynamic flux just on the panel containing the solar cells, limiting potential damage.

\section{A. Gauss pseudospectral method}

The solution to the previous problem is obtained using "collocation" or "direct approach". These methods discretize and transcribe the continuous-time optimization problem into a non-linear programing problem (NLP) that can be solved with dedicated NLP algorithms. A general minimization NLP problem can be stated as:

$$
\text { Minimze } f\left(x_{1}, x_{2}, \ldots, x_{n}\right)
$$

subject to:

$$
\begin{gathered}
g_{1}\left(x_{1}, x_{2}, \ldots, x_{n}\right) \leq b_{1} \\
\vdots \\
g_{m}\left(x_{1}, x_{2}, \ldots, x_{n}\right) \leq b_{m}
\end{gathered}
$$

where the functions $f$ and $g_{1}$ to $g_{m}$ are given linear or nonlinear functions. The problem is then, to select the values of the decision variables $\left(x_{1}, x_{2}, \ldots, x_{n}\right)$ from a given feasible region defined by $g_{1}$ to $g_{m}$, in order to minimize $f$, the cost function. To transform the continuous optimal control problem of Eq. 22 into an NLP we use the software GPOPS [29], a Matlab implementation of a Gauss pseudospectral direct collocation method. Both state and control are approximated by Lagrange interpolating polynomials, and the differential-algebraic equations are collocated at Legendre Gauss points. More information on this method, including its derivation, can be found in [19-21, 43, 44]. 
Due to the discretization, the result of the collocation is a large (but sparse) NLP, the solution of which is obtained with the sparse NLP solver provided in the NAG toolbox [30]. Other widely used software packages implementing PS methods for the solution of optimal control problems are DIDO [45] or the freely available tool PSOPT [46].

\section{Results}

The simulated orbit is a standard GTO with an initial perigee of $185 \mathrm{~km}$ (Table 2). Any higher perigee would be in general a less demanding scenario, with lower aerodynamic and gravity gradient torques. The attitude maneuver starts at a true anomaly value of -40 degrees and ends symmetrically at 40 degrees. These values correspond to an altitude of around $900 \mathrm{~km}$, where the aerodynamic torques start to be noticeable. Above this altitude the effects are normally neglected. During the perigee portion of the orbit we assume that the low thrust engine is off, so no other external torques are created and no attitude pointing requirements are needed. This could be either because the engine needs to be off as a consequence of the eclipse, or due to the optimization algorithm, or because it is decided to stop the thrust in order to facilitate the perigee pass. Therefore, the satellite can perform an attitude maneuver with no impact to the orbit. The parameters of the satellite are given in Tables 1 and 3, corresponding to a test full electric GEO satellite with deployed solar arrays at the beginning of an electric orbit raising. The satellite is equipped with reaction wheels, characterized by a maximum torque and storage capability (Table 3 ), the values representing typical reaction wheels for GEO platforms [47]. We assume that those values are equal for all axes.

The satellite starts the perigee passage with an initial attitude close to velocity pointing. After

Table 2: Initial orbit

\begin{tabular}{lcr}
\hline \hline & & Units \\
\hline True anomaly range & {$[-40,40]$} & deg \\
Apogee altitude & 35786 & $\mathrm{~km}$ \\
Perigee altitude & 185 & $\mathrm{~km}$ \\
Inclination & 28 & $\mathrm{deg}$ \\
\hline \hline
\end{tabular}


Table 3: Satellite actuator properties

\begin{tabular}{lcc}
\hline \hline & & Units \\
\hline$\tau_{\max }$ & 0.12 & N.m \\
$h_{\text {wax }}$ & 40 & N.m.s \\
$\Omega_{S A}$ & 0.5 & deg/s \\
\hline \hline
\end{tabular}

Table 4: Boundary values of the problem

\begin{tabular}{lccc}
\hline \hline & Initial & Final & Units \\
\hline $\boldsymbol{\sigma}$ & {$[0,-0.0733,0]$} & {$[0,-0.0733,0]$} & - \\
$\boldsymbol{\omega}$ & {$[0,0.0745,0]$} & {$[0,0.0745,0]$} & deg/s \\
$\boldsymbol{h}_{w}$ & {$[10 ;-5 ; 20]$} & {$[0,0,0]$} & N.m.s \\
$\boldsymbol{\delta}$ & {$[113.2,113.2]$} & {$[66.7,66.7]$} & deg \\
\hline \hline
\end{tabular}

the perigee passage, the spacecraft needs to be ready in firing configuration with again an attitude with the x-body aligned with the velocity vector (in this example). For both initial and final attitudes we assume no roll is needed. Boundary values of the problem are given in Table 4 . The wheels are assumed to have some initial amount of momentum and the final condition is zero for all axes, therefore, all the momentum needs to be dumped in the course of the maneuver. Initial and final values of solar array positions are calculated assuming that the right ascension of the apogee and the sun are the same. In addition, in order to simulate misalignments and real effects, a bias of 0.5 degrees is added to one of the arrays.

For comparison purposes, a simple non-optimized feedback eigen-axis controller is first implemented (Fig. 9). The result is a pitch inertial rotation. For the chosen reaction wheel properties, the aerodynamic torques exceed the control torque capability, resulting in a transient and uncontrolled attitude loss which is recovered once the density decreases. Therefore, the final pointing requirements at 40 degrees (perigee exit point) are not met. Also, from Fig. 9-b, the pure pitch maneuver saturates the momentum capability of reaction wheels (40 Nms per axis); in reality, attitude control is lost at that point. 

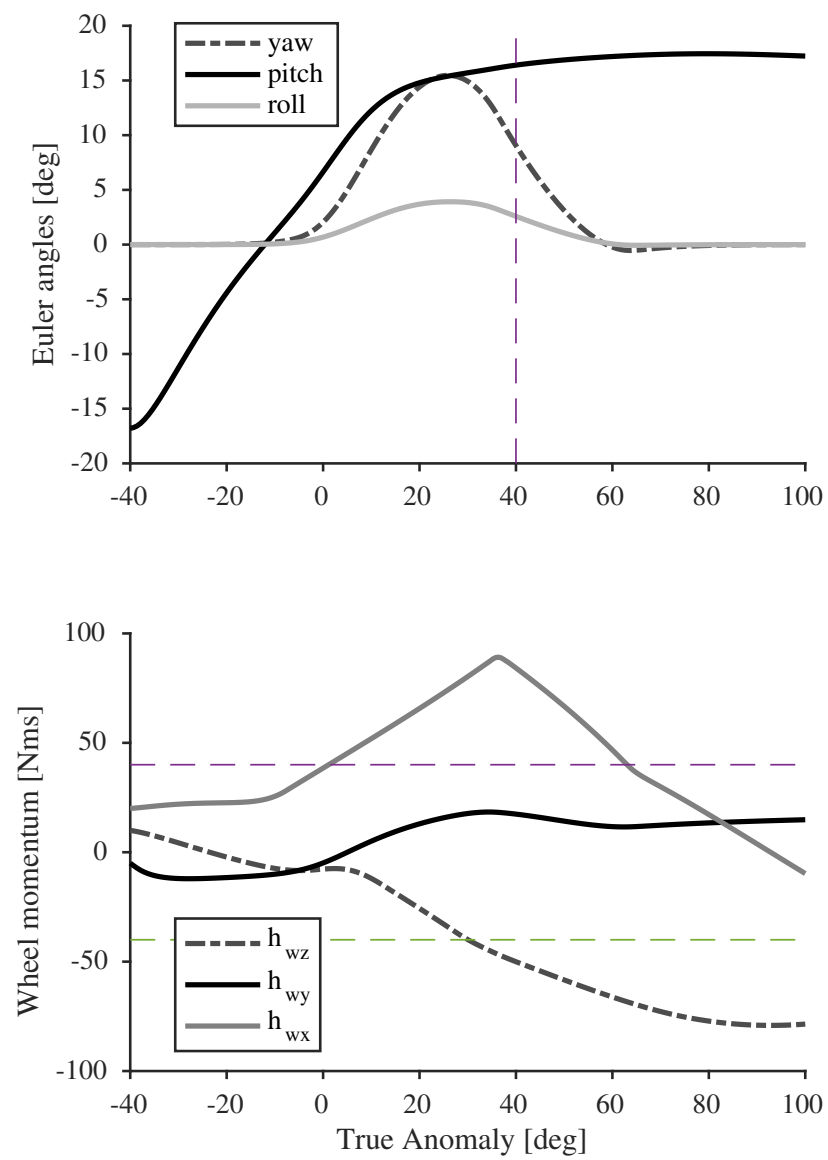

Fig. 9: Perigee maneuver performed with a non-optimized eigen-axis controller

\section{A. Fixed array rotation}

The optimization of the maneuver represented in Fig. 9 is shown in Fig. 10. The satellite performs an optimal 3-axis attitude maneuver that takes advantage of the environmental torques, not only to avoid wheel saturation but to dump all the initial wheel momentum (Fig. 10-b). The cost function for this problem is the integral of momentum magnitude during the manoeuver (Eq. 27) . All boundary conditions and constraints are satisfied. Fig. 10-b and Fig. 10-c show the resulting angular rates, and solar array positions, which are commanded at a fixed rate. Commanded wheel torque is presented in Fig. 11. The density variation during the maneuver together with the aerodynamic angles are shown in Fig. 12. Momentum in roll is controlled by means of the gravity gradient torque. An initial roll de-pointing is performed with the wheels, resulting in an increasing gravity gradient torque as the satellite approaches the perigee, this torque is used to dump the initial $h_{w x}$ momentum. In a similar way, pitch and yaw momentum are maintained within limits by 


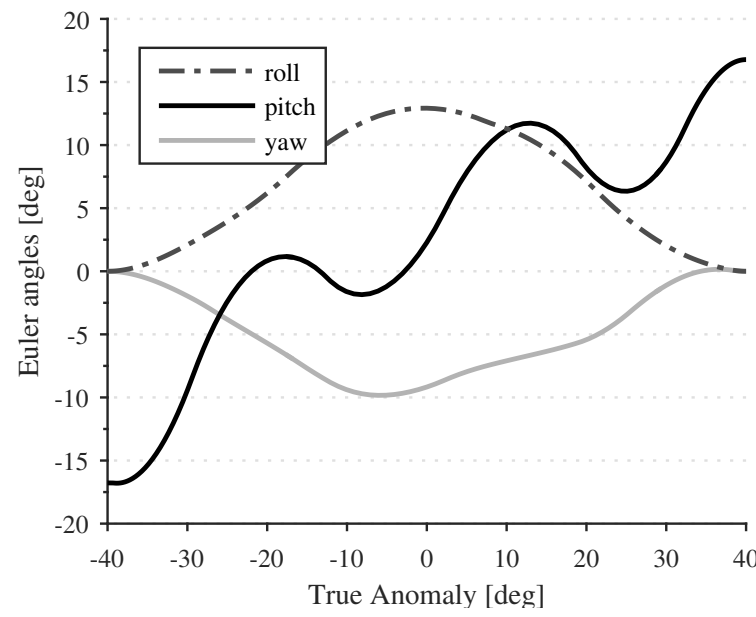

(a) Euler angles

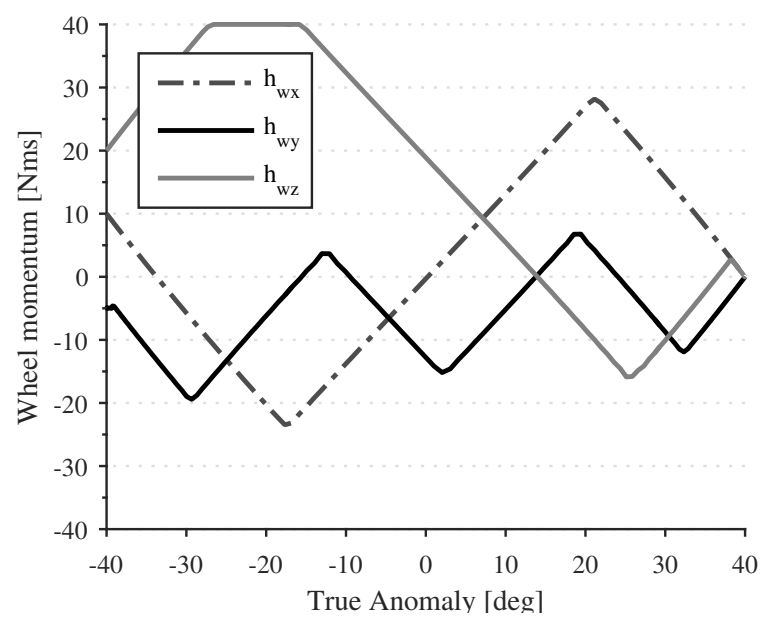

(c) Wheel momentum

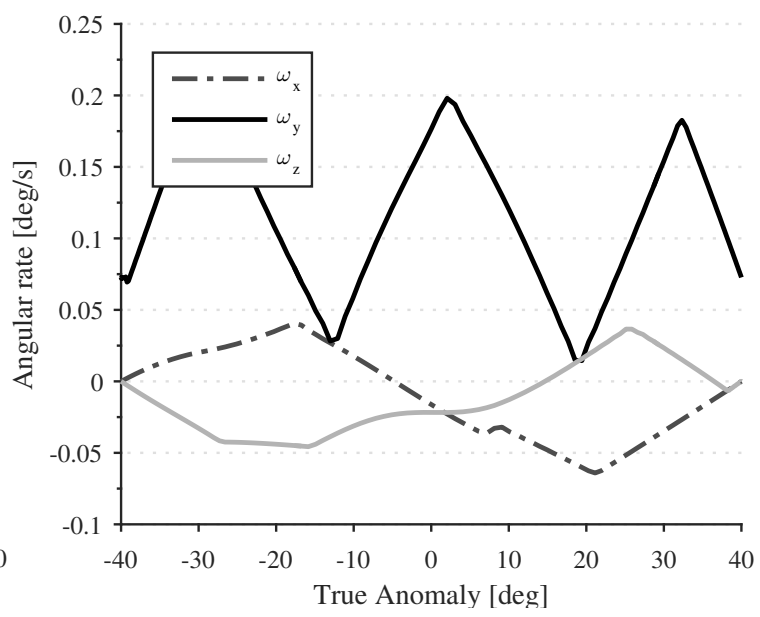

(b) Angular rates

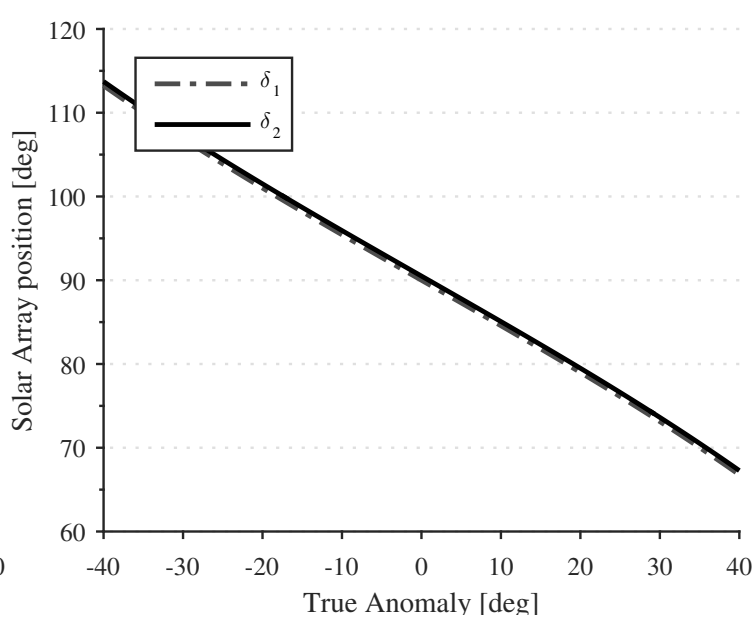

(d) Solar array positions

Fig. 10: Fixed array rotation.

means of aerodynamic torques.

The result of such a problem has been obtained by discretizing the cost function and the differential-algebraic equations using a mesh of 111 points. In order to facilitate convergence, an initial guess of only a few points is first produced. Then, the mesh is refined using as an initial guess the previously converged solution. Several initial conditions are tested to check that the same solution is obtained. The feasibility of the solution is verified as recommended in [45], by numerically propagating the initial state together with the solution from GPOPS, $u^{*}(t)$. The optimality of the solution is more difficult to check owing to the nature of the direct optimization methods. Some necessary (but not sufficient) conditions can be verified, as described in [45]. However, for this 

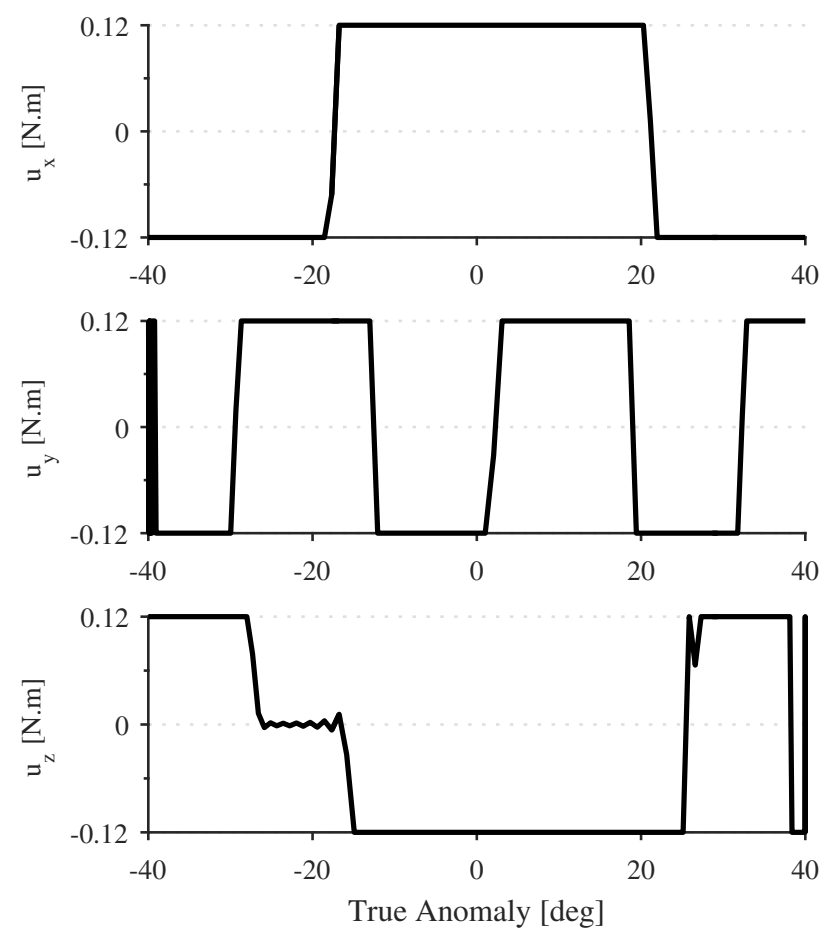

Fig. 11: Fixed array rotation. Optimal control torques applied to the wheels

problem, the optimality of the solution is not very important. As long as the solution is feasible and all the constraints are met, the maneuver can be performed, which is the main objective of the study. Notice that the cost function is not mass or time dependant; therefore, the potential existence of a solution with lower integral cost of accumulated wheel momentum does not modify any of the conclusions.

\section{B. Optimal solar array rotation}

Previously both solar arrays moved at a predefined fixed rate. However, the solar array positions can also be used to control and modulate aerodynamic torques. This can be achieved by introducing two extra control variables, rate of each solar array, as explained before. The resulting extra freedom allows better performance to be obtained or for more constraints to be introduced.

Fig. 13 presents the results of the maneuver with optimal solar array positions. An extra constraint has been also added, yaw de-pointing equal to zero. It can be observed how all the constraints are still met, yet with lower wheel requirements. The comparison with the fixed array rotation maneuver is shown in Fig. 14, where the evolution of the cost function, wheel momentum 

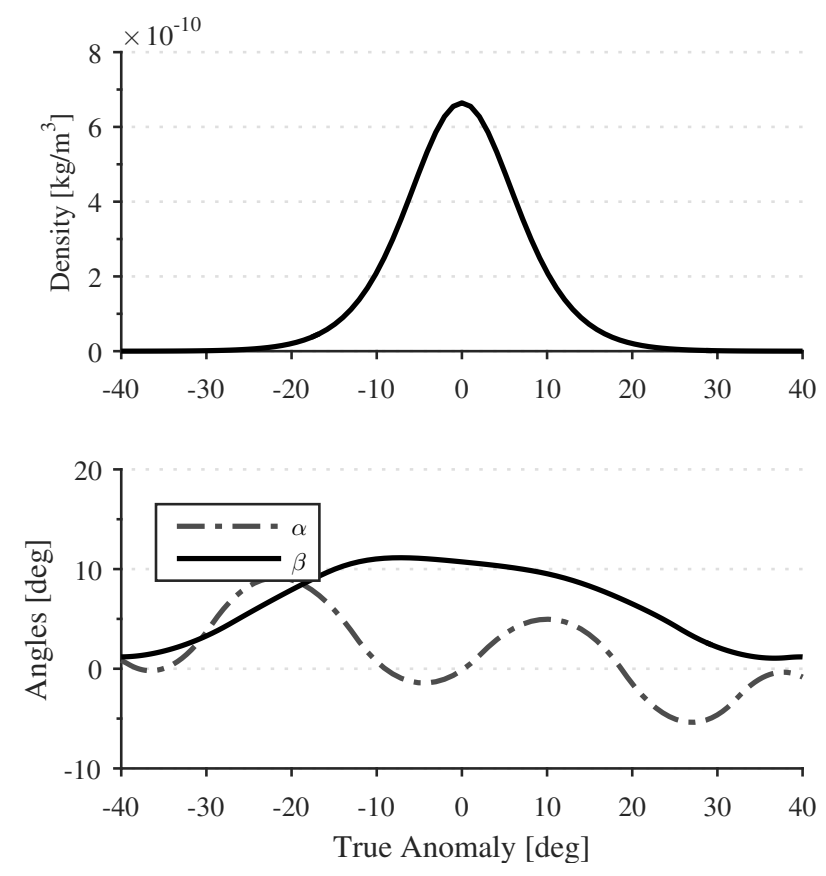

Fig. 12: Fixed array rotation. a) Density profile during the maneuver b) Aerodynamic angles

norm (Eq. 27), for both problems is presented.

The introduction of two new controls gives the problem a lot of flexibility. Therefore, different constraints or situations can be defined depending on spacecraft needs. For instance, previous cases assume that the electric propulsion thruster is off, consequently, there is no need to follow any predefined attitude path. This freedom in the attitude is used by the optimizer to meet maneuver constraints by means of an attitude maneuver, as highlighted in the fixed array rotation maneuver problem (Fig. 10). However, by adding these two new controls the aerodynamic torques can be controlled by means of the solar arrays and the attitude maneuver could be avoided.

This is useful if a certain attitude profile needs to be followed, for instance, in case the electric propulsion should remain on during the perigee passage.

This situation is presented in Fig 15, where no de-pointing with respect to LVLH is allowed, hence the satellite is at any moment earth pointed. The maneuver is still a pitch rotation, see the angular rate (Fig 15-b). Clearly, the combination of wheels and solar arrays is enough to perform the maneuver without wheel saturation in this case. The solar arrays are therefore used to minimize accumulated momentum during the maneuver but also to dump all the created momentum.

Following the same idea, we could also try to use angular de-pointing together with the solar 


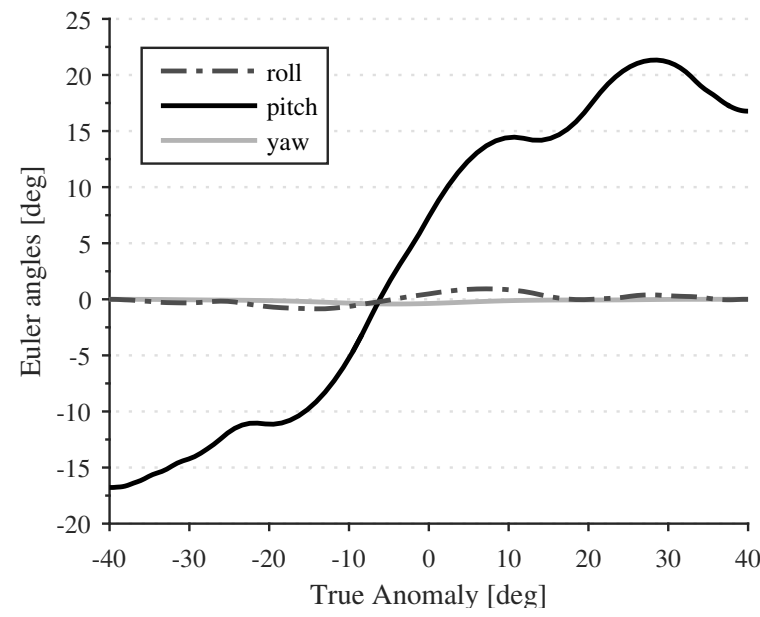

(a) Euler angles

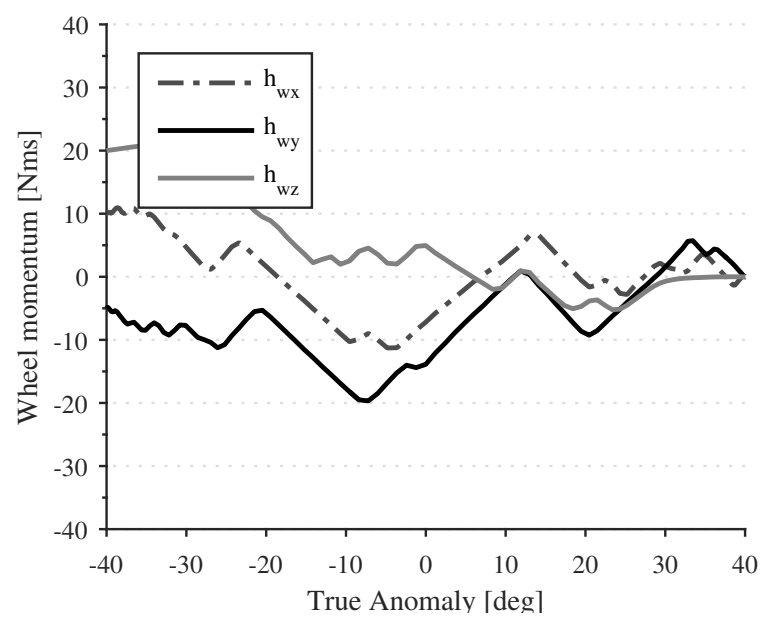

(c) Wheel momentum

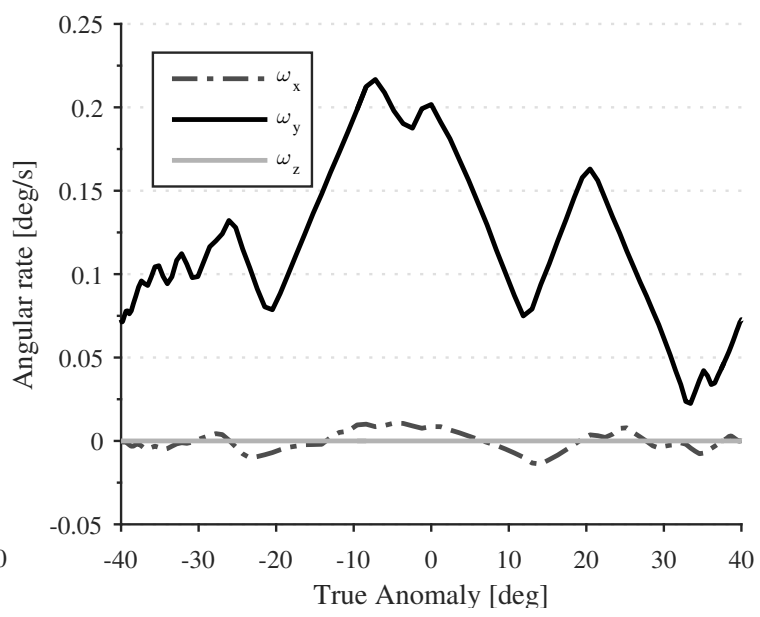

(b) Angular rates

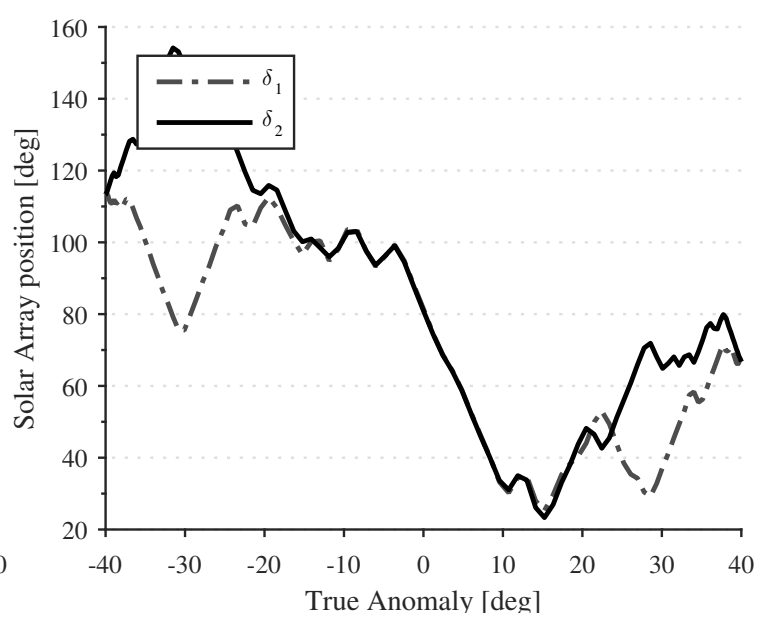

(d) Solar array positions

Fig. 13: Optimal solar array rotation.

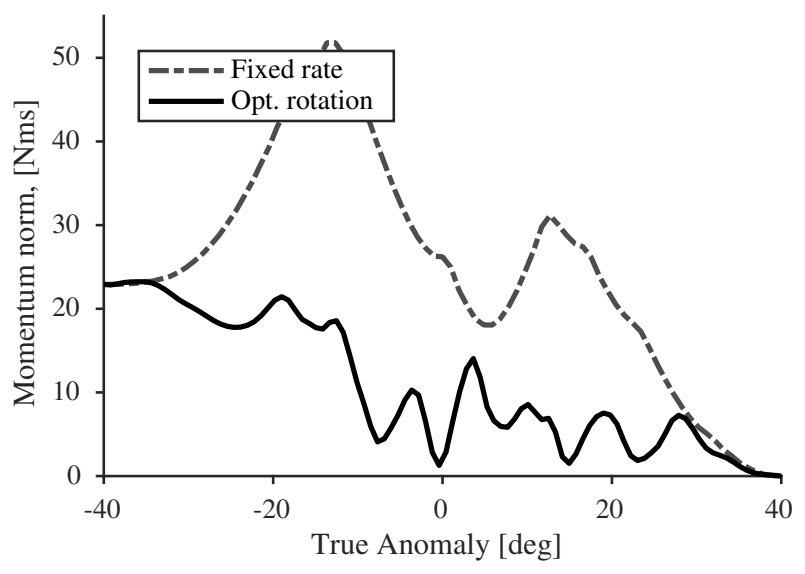

Fig. 14: Momentum norm comparison between fixed and optimized array rotation rates 


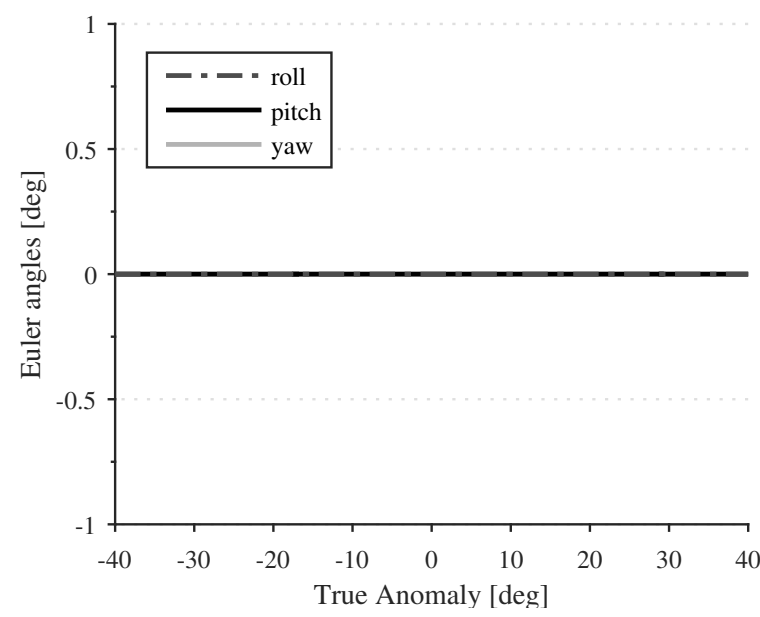

(a) Euler angles

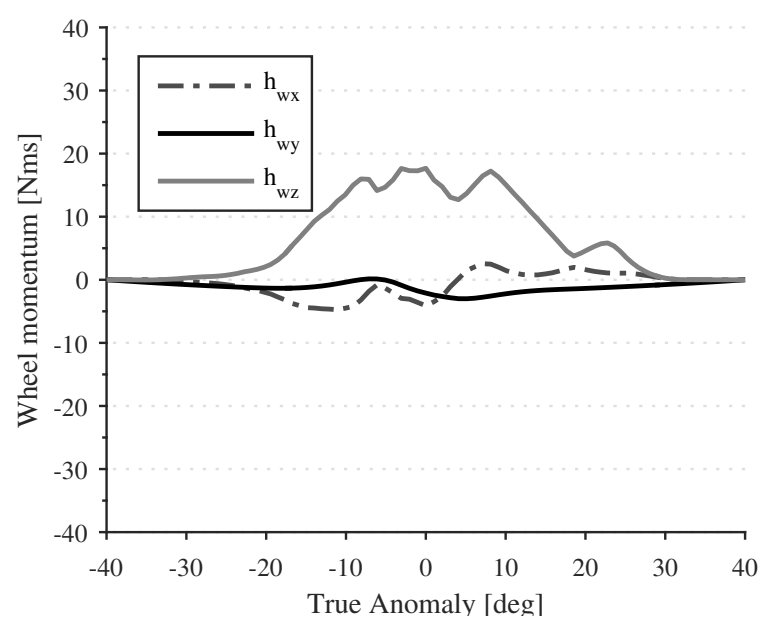

(c) Wheel momentum

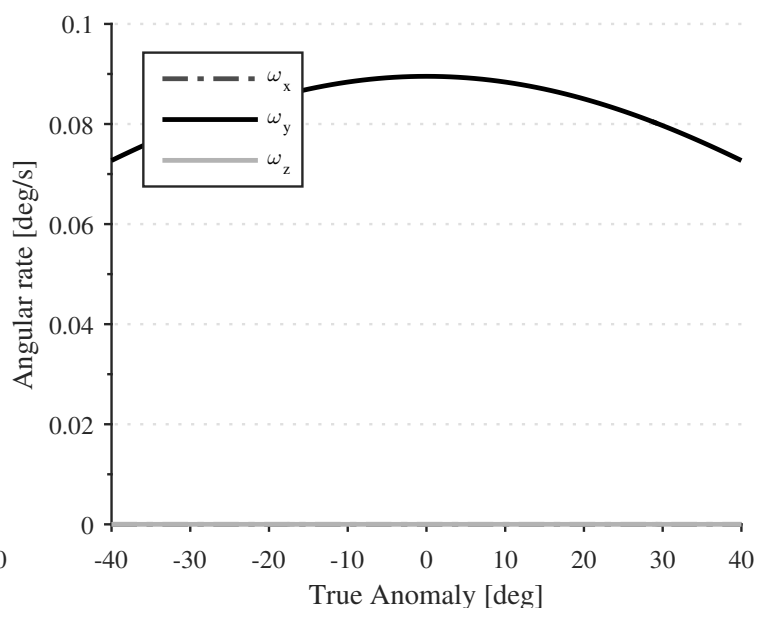

(b) Angular rates

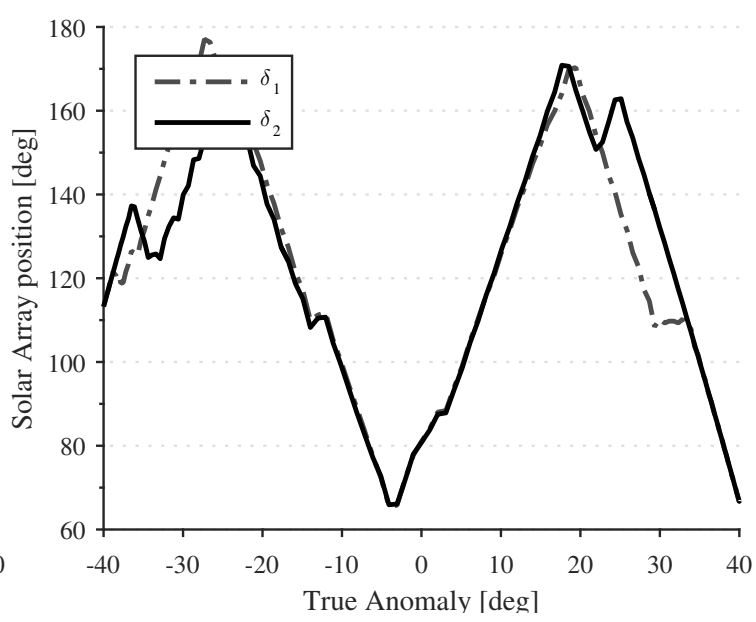

(d) Solar array positions

Fig. 15: Maneuver following a guided trajectory.

arrays to perform the maneuver. In such a case, no reaction wheels would be needed. The result is presented in Fig 16. Since there is no reaction wheel usage by definition, a different cost function is needed. We choose to minimize the aerodynamic flux on the solar arrays instead. Although the realization of the maneuver in such a way may seem unrealistic due to the intrinsic difficulties in the prediction of accurate aerodynamic torques and the fact that the torques created by the solar array rotations on the body have been neglected, it shows that it is possible to maneuver a satellite using just aerodynamic actuators and taking advantage of gravity gradient torques. This fact can be very useful in the case of an actuator failure.

In reality, a feedback control is needed in all cases, and any difference in the predicted momentum 


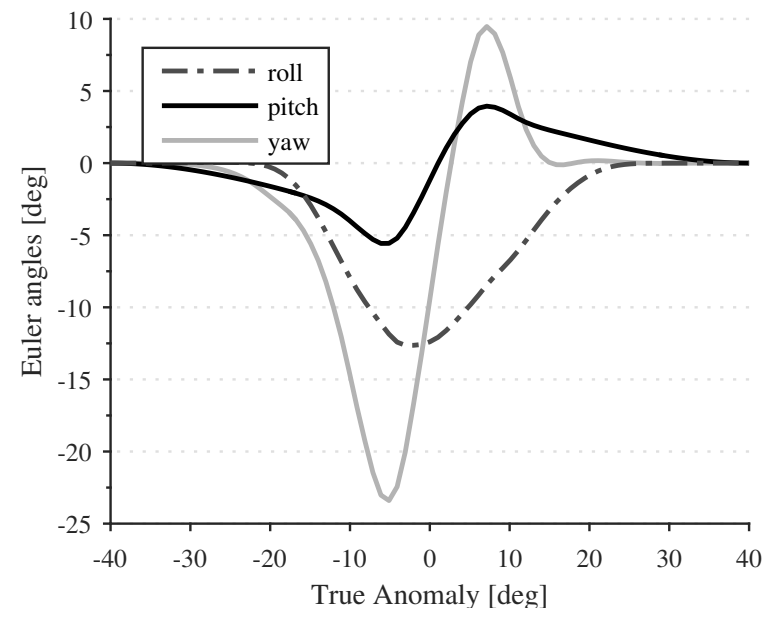

(a) Euler angles

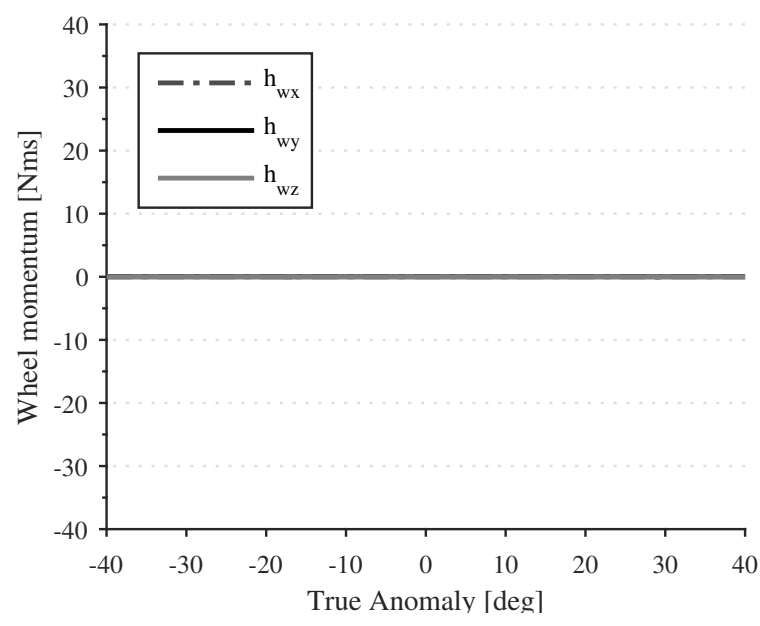

(c) Wheel momentum

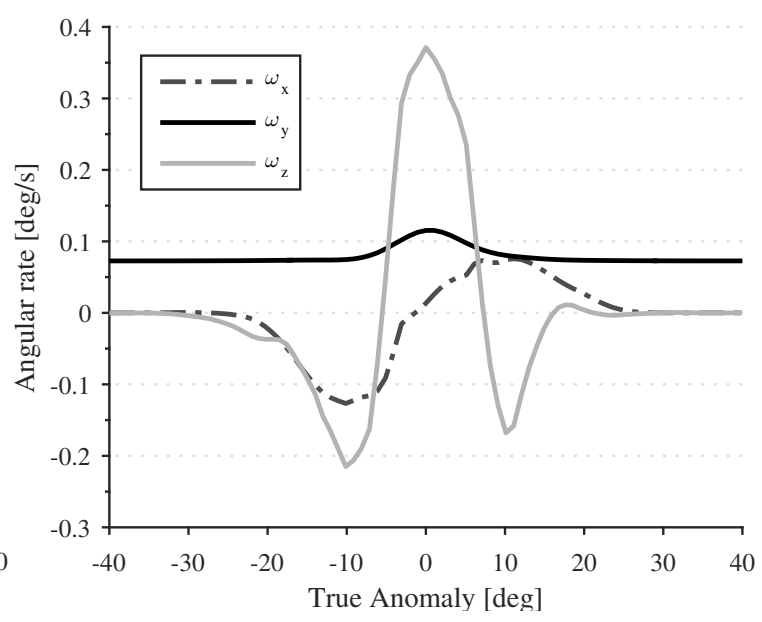

(b) Angular rates

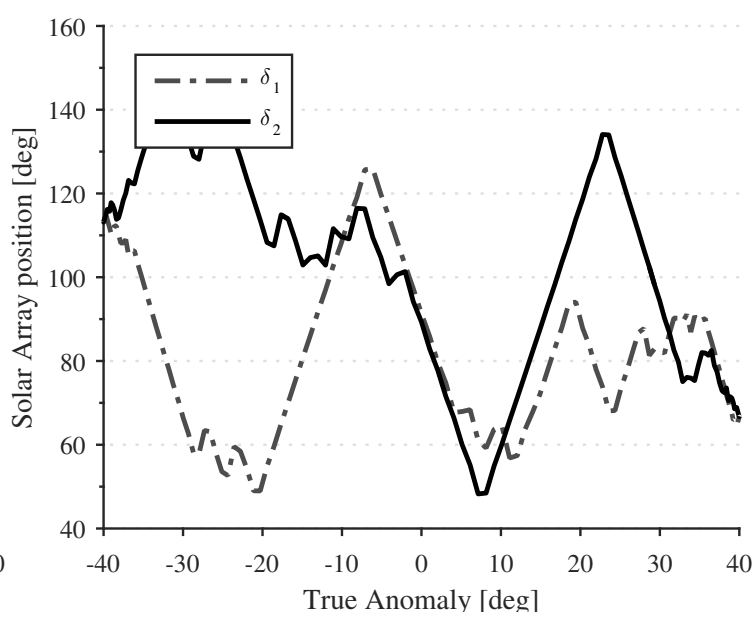

(d) Solar array positions

Fig. 16: Maneuver without reaction wheels.

would be absorbed by reaction wheels. The actual on-board implementation would follow the same principles as in [22-24]: the resulting optimal trajectory would be embedded in flight software and implemented as an open-loop guidance. Then, a closed-loop controller would follow the optimal attitude trajectory. In such a case, the predicted wheel speeds would deviate from reality as a result of non-modeled effects, therefore, appropriate margins are needed. A complete Monte Carlo analysis is required in order to design and validate the closed-loop controller. 


\section{Conclusions}

A method to facilitate the perigee passage of satellites under significant gravity and aerodynamic torques in geostationary transfer orbits (GTO) has been explored. Rather than bounding the magnitude of disturbance torques, a physically consistent modelling allows a more optimal maneuver to be performed while meeting the required constraints. To find the optimal path, a zero propellant maneuver (ZPM) problem for a non-circular orbit has been defined. The motion of solar arrays introduces extra degrees of freedom that can relax attitude hardware requirements or improve the maneuver performance. This attitude maneuver can be used to eliminate the mass penalties and cost associated with a perigee raising maneuver, whether by the satellite or the launch vehicle, or the need for a chemical thruster system. These mass and cost penalties can be significant, of the order of hundreds of kilograms, which could be used for additional payload or to significantly extend the operational life of the satellite. It can also be applied to scenarios where there is degraded attitude performance resulting from hardware failures.

\section{References}

[1] Svitak, A., "SpaceX Falcon 9 Launches First All-Electric Satellites," Aerospace Daily E Defense Report, Vol. 251, No. 39, 2015, p. 6.

[2] Svitak, A., "SES To Launch Two New Satellites On Falcon 9 Rockets," Aerospace Daily 8 Sefense Report, Vol. 251, No. 36, 2015, p. 8.

[3] Svitak, A., "SES Turns To Arianespace To Launch First Electric-Propulsion Spacecraft," Aerospace Daily $\& 3$ Defense Report, Vol. 251, No. 14, 2015, p. 6.

[4] Casaregola, C., "Electric Propulsion for Commercial Applications: In-Flight Experience and Perspective at Eutelsat," IEEE Transactions on Plasma Science, Vol. 43, No. 1, 2015, pp. 327-331, doi:10.1109/TPS.2014.2377782.

[5] Patton, C., "All Electric Satellites: Revolution or Evolution?" Via Satellite, Vol. 28, No. 5.

[6] Gonzalez del Amo, J., "European Space Agency Activities in Electric Propulsion," in "The 34th International Electric Propulsion Conference," Hyogo-Kobe, Japan, 2015.

[7] Svitak, A., "Dawn Of The All-Electric Satellite," Aerospace Daily $\&$ Defense Report, Vol. 251, No. 49, 2015, p. 3.

[8] Schäff, S. and Wiegand, A., "Advanced Electric Orbit-Raising Optimization for Operational Purpose," 
in "The 25th International Symposium on Space Flight Dynamics," Munich, 2015.

[9] Krier, G. and Mostaza-Prieto, D., "Fast and robust optimization of high fidelity continuous thrust transfer orbits with constraints," in "The 25th International Symposium on Space Flight Dynamics," Munich, 2015.

[10] Horne, R. B. and Pitchford, D., "Space Weather Concerns for All-Electric Propulsion Satellites," Space Weather, Vol. 13, No. 8, 2015, pp. 430-433, doi:10.1002/2015SW001198. 2015SW001198.

[11] SpaceX, Falcon 9 Launch Vehicle Payload User's Guide, 2015.

[12] United Launch Alliance, Delta IV Launch Services User's Guide, 2013.

[13] United Launch Alliance, Atlas V Launch Services User's Guide, 2010.

[14] Arianspace, Ariane 5 User's Manual, revision 1 ed., 2011.

[15] Meissinger, H., Dailey, C., and Valgore, M., "Optimal sun-alignment techniques of large solar arrays in electric propulsion spacecraft," in "International Electric Propulsion Conference," American Institute of Aeronautics and Astronautics, 1982, doi:10.2514/6.1982-1898. 0 .

[16] Meissinger, H., "A small, primary solar-electric propulsion demonstration satellite," in "SPACE Conferences and Exposition," American Institute of Aeronautics and Astronautics, 1992, doi:10.2514/6.1992-1566. 0 .

[17] Arianspace, Ariane 4 User's Manual, 1999.

[18] Bedrossian, N. S., Bhatt, S., Kang, W., and Ross, I. M., "Zero-propellant maneuver guidance," IEEE Control Systems, Vol. 29, No. 5, 2009, pp. 53-73, doi:10.1109/MCS.2009.934089.

[19] Fahroo, F. and Ross, I. M., "Costate Estimation by a Legendre Pseudospectral Method," Journal of Guidance, Control, and Dynamics, Vol. 24, No. 2, 2001, pp. 270-277, doi:10.2514/2.4709.

[20] Fahroo, F. and Ross, I. M., "Direct Trajectory Optimization by a Chebyshev Pseudospectral Method," Journal of Guidance, Control, and Dynamics, Vol. 25, No. 1, 2002, pp. 160-166, doi:10.2514/2.4862.

[21] Benson, D., A Gauss Pseudospectral Transcription for Optimal Control, Ph.D. thesis, Massachusetts Institute of Technology. Dept. of Aeronautics and Astronautics, 2005.

[22] Bedrossian, N., Bhatt, S., Lammers, M., Nguyen, L., and Zhang, Y., "First Ever Flight Demonstration of Zero Propellant Maneuver(TM) Attitute Control Concept," in "AIAA Guidance, Navigation and 
Control Conference and Exhibit," American Institute of Aeronautics and Astronautics (AIAA), 2007, doi:10.2514/6.2007-6734.

[23] Bedrossian, N. and Bhatt, S., "Space station Zero-Propellant Maneuver guidance trajectories compared to eigenaxis," in "2008 American Control Conference," , 2008, pp. 4833-4838, doi:10.1109/ACC.2008.4587259.

[24] Bhatt, S., Bedrossian, N., and Nguyen, L., "Optimal Propellant Maneuver Flight Demonstrations on ISS," in "AIAA Guidance, Navigation, and Control (GNC) Conference," American Institute of Aeronautics and Astronautics (AIAA), 2013, doi:10.2514/6.2013-5027.

[25] Fahroo, F. and Ross, I. M., "Advances in Pseudospectral Methods for Optimal Control," in "AIAA Guidance, Navigation and Control Conference and Exhibit," American Institute of Aeronautics and Astronautics (AIAA), 2008, doi:10.2514/6.2008-7309.

[26] Karpenko, M., Bhatt, S., Bedrossian, N., Fleming, A., and Ross, I. M., "First Flight Results on TimeOptimal Spacecraft Slews," Journal of Guidance, Control, and Dynamics, Vol. 35, No. 2, 2012, pp. $367-376$, doi:10.2514/1.54937.

[27] Karpenko, M., Bhatt, S., Bedrossian, N., and Ross, I. M., "Flight Implementation of Shortest-Time Maneuvers for Imaging Satellites," Journal of Guidance, Control, and Dynamics, Vol. 37, No. 4, 2014, pp. 1069-1079, doi:10.2514/1.62867.

[28] Kedare, S. and Ulrich, S., "Formulation of Torque-Optimal Guidance Trajectories for a CubeSat with Degraded Reaction Wheels," in "AIAA Guidance, Navigation, and Control Conference," American Institute of Aeronautics and Astronautics (AIAA), 2016, doi:10.2514/6.2016-0088.

[29] Rao, A. V., Benson, D. A., Huntington, G. T., and Francolin, C., User's Manual for GPOPS: a MATLAB package for dynamic optimization using the Gauss pseudospectral method, University of Florida report, 2008.

[30] The Numerical Algorithms Group (NAG), Oxford, United Kingdom, www.nag.com, The NAG Toolbox for MATLAB囚.

[31] Tsiotras, P. and Longuski, J. M., "A new parameterization of the attitude kinematics," Journal of the Astronautical Sciences, Vol. 43, No. 3, 1995, pp. 243-262. 
[32] Schaub, H. and Junkins, J. L., "Stereographic orientation parameters for attitude dynamics: A generalization of the Rodrigues parameters," Journal of the Astronautical Sciences, Vol. 44, No. 1, 1996, pp. 1-19.

[33] Sidi, M. J., Spacecraft Dynamics and Control: A Practical Engineering Approach (Cambridge Aerospace Series), Cambridge University Press, 2000.

[34] Picone, J. M., Hedin, A. E., Drob, D. P., and Aikin, A. C., "NRLMSISE-00 empirical model of the atmosphere: Statistical comparisons and scientific issues," Journal of Geophysical Research: Space Physics, Vol. 107, No. A12, 2002, pp. SIA 15-1-SIA 15-16, doi:10.1029/2002JA009430. 1468.

[35] Bowman, B., Tobiska, W. K., Marcos, F., Huang, C., Lin, C., and Burke, W., "A New Empirical Thermospheric Density Model JB2008 Using New Solar and Geomagnetic Indices,” in “AIAA/AAS Astrodynamics Specialist Conference and Exhibit," American Institute of Aeronautics and Astronautics (AIAA), 2008, doi:10.2514/6.2008-6438.

[36] Drob, D. P., Emmert, J. T., Crowley, G., Picone, J. M., Shepherd, G. G., Skinner, W., Hays, P., Niciejewski, R. J., Larsen, M., She, C. Y., Meriwether, J. W., Hernandez, G., Jarvis, M. J., Sipler, D. P., Tepley, C. A., O’Brien, M. S., Bowman, J. R., Wu, Q., Murayama, Y., Kawamura, S., Reid, I. M., and Vincent, R. A., "An empirical model of the Earth's horizontal wind fields: HWM07," Journal of Geophysical Research: Space Physics, Vol. 113, No. A12, doi:10.1029/2008JA013668. A12304.

[37] Mostaza Prieto, D., Graziano, B. P., and Roberts, P. C., "Spacecraft drag modelling," Progress in Aerospace Sciences, Vol. 64, 2014, pp. 56-65, doi:10.1016/j.paerosci.2013.09.001.

[38] Wachman, H. Y., "The Thermal Accommodation Coefficient: A Critical Survey," ARS Journal, Vol. 32, No. 1, 1962, pp. 2-12, doi:10.2514/8.5939.

[39] Moe, K. and Moe, M. M., "Gas-surface interactions and satellite drag coefficients," Planetary and Space Science, Vol. 53, No. 8, 2005, pp. 793-801, doi:10.1016/j.pss.2005.03.005.

[40] Sentman, L. H., "Free molecular flow theory and its application to the determination of aerodynamic forces Rep,” Tech. Rep. LMSC-448514, Lockheed Missiles and Space Co.,, Sunnyvale, CA, 1961.

[41] Walker, A., Mehta, P., and Koller, J., "Drag Coefficient Model Using the Cercignani-Lampis-Lord 
Gas-Surface Interaction Model," Journal of Spacecraft and Rockets, Vol. 51, No. 5, 2014, pp. 15441563

doi:10.2514/1.a32677.

[42] Bird, G. A., Molecular Gas Dynamics and the Direct Simulation of Gas Flows (Oxford Engineering Science Series), Clarendon Press, 1994.

[43] Huntington, G. T., Advancement and analysis of a Gauss pseudospectral transcription for optimal control problems, Ph.D. thesis, Massachusetts Institute of Technology. Dept. of Aeronautics and Astronautics, 2007.

[44] Garg, D., "An overview of three pseudospectral methods for the numerical solution of optimal control problems," in "Advances in the Astronautical Sciences," AAS/AIAA Astrodynamics Specialist Conference, Pittsburgh, Pennsylvania, Vol. 135, 2009, pp. 475-487.

[45] Ross, I. M., “A Beginner's Guide to DIDO: A MATLAB Application Package for Solving Optimal Control Problems," Tech. Rep. TR-711, Elissar, LLC, 2007.

[46] Becerra, V. M., "Solving complex optimal control problems at no cost with PSOPT," in "IEEE Multiconference on Systems and Control," IEEE, Yokohama, Japan, 2010, pp. 1391-1396.

[47] Marshall, T. and Fletcher, M., "ACTUATORS: Meeting high-quality RWA commercial demand through innovative design," in Danesy, D., ed., "8th European Space Mechanisms and Tribology Symposium," Vol. 438 of ESA Special Publication, 1999, p. 253. 